\title{
Planification urbaine et mutations économiques. L'arrondissement de la baie à la croisée des chemins
}

\author{
Martin Simard et Carl Brisson \\ Université du Québec à Chicoutimi
}

\section{Introduction}

Au Canada et au Québec, bon nombre de villes, petites et moyennes, positionnées en milieu périphérique, vivent des difficultés sur les plans économique et démographique dans le cadre de la mondialisation des marchés et de l'émergence d'une économie du savoir. Les analystes les plus pessimistes entrevoient la stagnation ou le déclin progressif pour ces ensembles urbains de taille modeste à l'économie généralement peu diversifiée. Le défi d'assurer la vitalité économique, de maintenir une gamme étendue de services publics et privés de même que d'offrir un cadre urbain de qualité est fort exigeant pour les dirigeants en fonction. L'image négative du milieu, la faiblesse des ressources humaines et financières ainsi que le conservatisme ambiant peuvent constituer

L'absence de planification urbaine adaptée à la réalité des années $\mathbf{2 0 0 0}$ se fait fortement sentir. des freins importants à l'innovation et au développement. La mise en place d'initiatives locales ou de projets de développement apparaît alors être un élément déterminant pour l'avenir collectif.

L'arrondissement de La Baie à Saguenay est un exemple de milieu périphérique frappé par des difficultés de tout ordre. Du point de vue socioéconomique, la communauté locale a été gravement touchée. En effet, l'un des piliers de l'activité industrielle, soit l'usine de papier de l'Abitibi-Consolidated (ACI), a fermé ses portes en 2003, occasionnant la perte de 650 emplois. Par la suite, une scierie a cessé ses activités, ce qui s'est traduit par la perte d'une centaine d'emplois. Certains secteurs de l'activité commerciale ont également vécu un déclin et l'on assiste au départ de certains services administratifs et financiers vers d'autres arrondissements. Sur le plan démographique, l'arrondissement de La Baie subit une décroissance après avoir connu une hausse continue de sa population qui atteignait 21000 habitants en 1996. Cette décroissance s'accompagne d'une modification de la structure par âge de la population.

Dans ce contexte, l'absence de planification urbaine adaptée à la réalité des années 2000 se fait fortement sentir. Ainsi, c'est le plan d'urbanisme de l'ex-ville de La Baie en vigueur depuis 1991 qui est toujours en fonction malgré le regroupement qui a créé la Ville de Saguenay en 2002. Cet outil n'est plus adapté à la réalité du milieu. De plus, on assiste depuis quelques années à un redéploiement spatial de plusieurs activités dans l'arrondissement de $\mathrm{La}$ Baie. En conséquence, il apparaît primordial de se pencher sur les enjeux d'aménagement et les projets de développement actuels à La Baie. Cet arrondissement se révèle être à la croisée des chemins si l'on considère les changements récents à la structure industrielle, la superficie et la localisation des terrains devenus vacants ainsi que l'ampleur économique et urbanistique des projets à l'étude ou en voie d'implantation.

À l'intérieur de ce texte, nous traiterons de trois enjeux d'aménagement qui prennent la forme de projets de développement. Nous utiliserons la méthode de l'étude de cas et puiserons nos informations dans divers documents d'urbanisme, des coupures de presse et des visites de terrain. Ces projets situés sur le territoire de La Baie sont les suivants : le terminal de croisières sur le quai Agésilas-Lepage 
à Bagotville, la réutilisation du site d'ACI à PortAlfred et l'implantation d'un terminal méthanier au port de Grande-Anse. Au préalable, nous tracerons un bref portrait de l'arrondissement de La Baie afin de comprendre l'environnement dans lequel devraient s'insérer ces interventions.

\section{L'ARRONDISSEMENT DE LA BAIE : UN BREF PORTRAIT}

Le paysage de La Baie est façonné par la présence de la baie des Ha! Ha! et par la dualité entre les basses-terres en bordure de la baie et le plateau du Haut-Saguenay. Ces deux unités physiographiques sont séparées par des dénivellations entrecoupées des rivières à Mars et $\mathrm{Ha}$ ! Ha! et d'une série de ravins. Le territoire de la Baie des Ha! Ha ! a été ouvert à la colonisation en 1838 et il fut relié au réseau ferroviaire en 1911, après Roberval (1888) et Chicoutimi (1893). Cependant, il faut attendre l'arrivée de l'industrie pour que le secteur se développe pleinement. Tirant profit de la première guerre mondiale, J.E.A. Dubuc et ses partenaires anglais se lancent dans la production de pâte chimique à Port-Alfred. La construction de l'usine, d'infrastructures portuaires et ferroviaires et d'un établissement urbain débuta en 1915.

Sur le plan administratif, la mise en place des municipalités sur ce territoire correspond à une succession de morcellement du Canton de Bagot, une municipalité ayant eu un vaste territoire, vers de plus petites entités. Ce processus qui s'échelonna de 1848 à 1920 fut suivi d'une période de regroupements municipaux qui débuta en 1953. Par ailleurs, 1976 et 2002 constituent des dates clefs, soit les moments de fondation des villes de La Baie et de Saguenay. Le morcellement administratif qu'a longtemps connu l'arrondissement de La Baie reflète, dans une large mesure, la structure du peuplement qui est divisée historiquement en trois noyaux urbains, soit Grande-Baie, Port-Alfred et Bagotville, sans compter les zones rurales d'habitats.

$\mathrm{Au}$ cours des années 1940, La Baie connaîtra un accroissement significatif de sa population et une augmentation sensible des conditions de vie de la population. C'est aussi à cette époque que le gouvernement canadien établit une base militaire à Ba- gotville (1942). Néanmoins, l'absence de planification urbaine dans un contexte de croissance est susceptible d'entraîner des conflits et des disfonctionnements. Par exemple, les secteurs urbanisés ne semblent plus en mesure d'absorber le développement urbain à partir du milieu du siècle. L'utilisation de plus en plus massive de l'automobile, l'accès aux résidences unifamiliales, l'arrivée des grandes chaînes de magasins, tous ces phénomènes se conjuguent pour faire éclater les limites des périmètres urbains. À ce moment, les contraintes topographiques et l'exiguïté des territoires municipaux de Bagotville et de Port-Alfred rendent pratiquement impossible l'expansion résidentielle et industrielle.

\section{L'absence de planification urbaine dans un contexte de croissance est susceptible d'entraîner des conflits et des disfonction- nements.}

En 1977, la nouvelle Ville de La Baie adopte son premier plan d'urbanisme. La mise en place de ce nouvel outil, tout en cherchant à atténuer les rivalités, ne remet pas en cause les trois secteurs traditionnels que sont Bagotville, Port-Alfred et GrandeBaie. Ainsi, il n'y a pas de centre-ville formellement identifié. Toute la planification s'articule autour d'une amélioration des liens tant physiques que socio-économiques entre les secteurs afin d'accentuer les échanges. Chaque secteur aura un rôle spécialisé: Port-Alfred poursuivra son essor industriel, Bagotville consolidera son dynamisme commercial alors que Grande-Baie maintiendra son caractère domiciliaire. ${ }^{1}$ Le concept proposé en 1977 est grandement influencé par le potentiel portuaire existant sur le territoire de la ville. Toutefois, le développement touristique ne fait pas partie de la stratégie d'aménagement, l'emphase est mise sur la récréation. Malgré tous les efforts entrepris, les structures industrielle et commerciale de La Baie demeurent fragiles et une crise frappe la communauté au début des années 2000. 


\section{LES ENJEUX D'AMÉNAGEMENT ACTUELS}

\section{Le terminal de croisières sur le quai Agésilas- Lepage à Bagotville}

Le premier des trois projets analysés dans le cadre de cet article est celui du terminal de croisières sur le quai Agésilas-Lepage à Bagotville (voir la figure 1). L'idée de ce terminal a été lancée en 2003 à la suite de discussions entre les élus de la Ville de Saguenay et les administrateurs du Port de Québec, organisme sous juridiction fédérale. Il s'agit d'amener les navires de croisières qui visitent le Saint-Laurent et ses affluents à partir de la ville de Québec à venir jusqu'à la Baie des $\mathrm{Ha}$ ! Ha! pour y faire escale un ou deux jours. Cette industrie en croissance pourrait stimuler l'économie locale et consolider les activités touristiques, notamment à la fin de l'été et en automne. Le projet apparaît fort alléchant dans le contexte des difficultés économiques baieriveraines avec des retombées économiques de 45,7 millions de dollars prévues pour les cinq premières années d'opération. Néanmoins, ce projet ambitieux connaît de nombreux rebondissements et suscite la controverse, notamment pour l'ampleur des investissements requis.
En 2004, s'enclenche la réflexion sur la localisation d'un quai d'escale par "Promotion Saguenay », institution parapublique gérée directement par le maire de Saguenay. Le site choisit pour l'infrastructure d'accueil est le quai AgésilasLepage, lieu situé dans la partie nord du centre-ville de Bagotville qui pourrait permettre une intervention de revitalisation urbaine. L'utilisation des installations portuaires d'Alcan à La Baie a aussi été considérée mais la manutention industrielle qui inclut des produits dangereux commanderait des mesures de sécurité élargies pour permettre l'accueil de croisiéristes. D'autre part, les principes qui régissent la navigation commerciale font en sorte que les navires sont desservis selon leur ordre d'arrivée. Une procédure incompatible avec l'exploitation de croisières, les exploitants devant obtenir confirmation d'une place à quai plus d'un an à l'avance. Le quai Agésilas-Lepage est d'ailleurs temporairement aménagé pour permettre la venue de navires à titre exploratoire en 2005 et en 2006. Cette expérience a suscité un certain enthousiasme auprès de la population et des intervenants économiques locaux.

Figure 1

Les projets de développement à La Baie

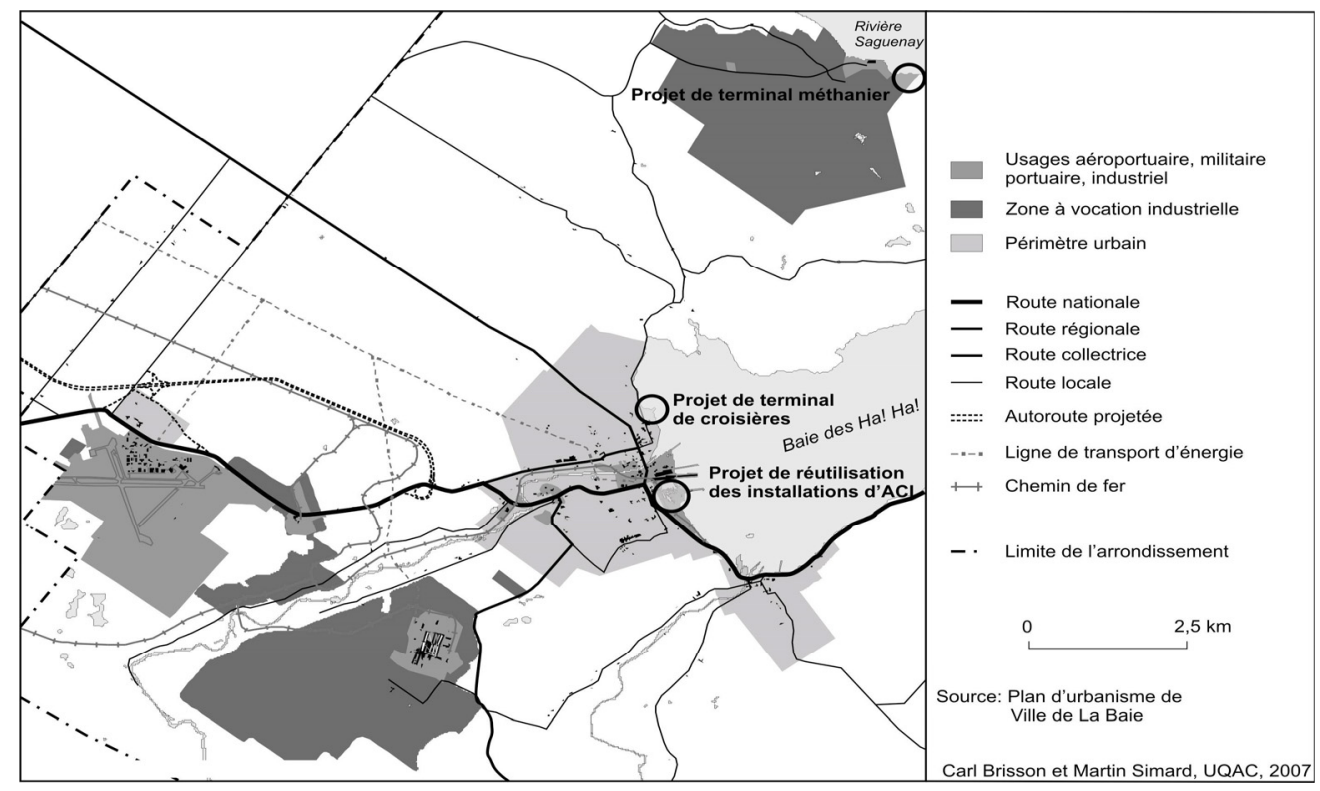

Sur le plan du processus, une étude d'impact a été déposée en 2005 au Bureau d'audiences publiques en environnement (BAPE) afin de contribuer à une démarche décisionnelle éclairée. Cependant, le 11 mai 2006, le maire de Saguenay annonce qu'il a demandé au ministère de Développement durable, 
de l'environnement et de la faune de suspendre les procédures d'analyse du BAPE. Celles-ci sont finalement reprises, avec une séance d'information le 11 décembre, mais il n'y aura pas de demande formelle d'audiences publiques. Pour sa part, la Ville et Promotion Saguenay organiseront des rencontres d'information dans les trois arrondissements, les 5, 6 et 7 décembre $2006 .^{2}$ Il y aura également ouverture des registres dans l'arrondissement La Baie, en vue de la possible tenue d'un référendum sur un règlement d'emprunt de 11 millions de dollars, les 19 , 20 et 21 décembre 2006. Celui-ci n'aura pas lieu, le nombre de signatures requis n'ayant pas été atteint. Le 17 février de la même année, les gouvernements supérieurs ont annoncé des appuis financiers respectifs de 9,8 millions de dollars.

L'arrondissement de La Baie, comme plusieurs localités industrielles, doit composer avec un milieu urbain en recomposition. Les croisières constituent une activité touristique susceptible de bien s'intégrer à une stratégie de relance économique et d'amélioration du cadre de vie. En ce qui concerne l'intégration du quai d'escale et d'un futur « village portuaire » à l'espace urbain du centre-ville de Bagotville, des terrains ont été acquis par la Ville de gré à gré et un mandat a été consenti à des consultants dans le but de concevoir un plan d'intervention. Y aura-t-il un contrôle architectural pour les nouvelles constructions et la rénovation des bâtiments existants? Ce programme sera-t-il accompagné d'incitatifs (réduction de taxes, programme de rénovation, etc.) visant la venue de nouveaux commerces dans le centre-ville ? Ces questions demeurent ouvertes pour le moment!

\section{Les croisières constituent une activité touris- tique susceptible de bien s'intégrer à une stratégie de relance économique et d'amélioration du cadre de vie.}

\section{La réutilisation des installations d'ACI à Port-} Alfred

Les terrains et les bâtiments d'Abitibi-Consolidated sont situés sur les rives de La Baie des Ha! Ha! Ceux-ci jouxtent le site des installations portuaires et ferroviaires d'ALCAN qui est localisé en bordure de la Rivière-à-Mars. Cet ensemble industriel posi- tionné au cœur de l'arrondissement domine le paysage et l'économie depuis des décennies. Le terrain industriel, qui possède une topographie généralement plane, se retrouve à l'interface d'un environnement urbain et d'un environnement maritime. En effet, le terrain est bordé au sud par le boulevard de la Grande-Baie-Sud et, à l'ouest, au-delà de la voie ferrée, par la $1^{\text {re }}$ rue et l'avenue du Port où subsiste difficilement une aire commerciale. En ce qui concerne la réglementation de zonage, le terrain de l'usine est évidemment affecté à l'activité industrielle.

Bien que les deux propriétés industrielles voisines soient maintenant gérées de manière distincte et qu'elles connaissent un sort bien différent, leur évolution s'est effectuée en parallèle. Il appert donc de discuter brièvement des équipements d'ALCAN avant de nous attarder au destin de l'usine et du site d'ACI. À la suite de la construction de l'usine de la Ha ! Ha ! Bay Sulfite en 1918, le quai existant depuis quelques années est rallongé, élargi et doté de quatre voies ferrées en 1920. ${ }^{3}$ En 1924, l'entrepreneur chicoutimien Dubuc, qui en est le propriétaire, est acculé à la faillite. La Compagnie ALCOA fait alors l'acquisition des actifs de la Compagnie générale du port de Chicoutimi et de la compagnie de chemin de fer Roberval-Saguenay. Cet achat permet aujourd'hui à Alcan d'alimenter en matières premières son complexe d'Arvida à partir de ses deux quais de La Baie. ${ }^{4}$

Le site sur lequel a été érigée l'usine de Port-Alfred se situe sur les lots 1982, 1983, 1984, 1896, et 1899 du cadastre officiel de la Ville de La Baie. Il est d'une superficie de 38,885 ha. Cette superficie se retrouve en partie sur la terre ferme (26,965 ha.) et en partie sous l'eau (11,960 ha). ${ }^{5}$ Au moment de la fermeture définitive d'ACI, on observait sur ce terrain 22 réservoirs destinés à contenir de la pâte à papier, du mazout, divers produits chimiques nécessaires à la production du papier, de l'huile et des eaux usées en traitement. Quelque 81 bâtisses, séparées ou contiguës, servant à abriter les divers équipements de l'usine, et un convoyeur (inutilisé depuis 25 ans) se remarquaient aussi sur l'emprise foncière. D'autre part, le quai de déchargement des billes de bois qui n'est plus utilisé depuis l'arrêt du flottage du bois en 1990 est situé sur la partie ennoyée du site. 
En 2005, l'usine a cessé définitivement ses activités. Un an plus tard, l'usine et la majorité des équipements furent démolis. Ainsi, un symbole important de l'activité industrielle baieriveraine disparaît du paysage. En 2006, Abitibi Consolidated cède à l'entreprise Biomatera environ $40 \%$ du terrain rendu disponible par la démolition de sa papetière de Port-Alfred. L'entreprise de production de plastique biodégradable devrait s'installer dans l'un des rares bâtiments qui évitera le pic des démolisseurs. L'autre partie du terrain $(60 \%)$ a été cédée à la Ville de Saguenay contre la somme d'un dollar. ${ }^{6}$ Quelle vocation donner à ce site de grande dimension qui offre une vue magnifique sur la Baie? Comment coordonner l'aménagement de ces terrains avec l'intervention prévue dans le secteur du quai Agésilas-Lepage? Doit-on privatiser cet espace ou y maintenir une fonction communautaire ? Ces interrogations restent toujours sans réponse au début de l'année 2007.

\section{Quelle vocation donner à ce site de grande dimension qui offre une vue magnifique sur la Baie?}

\section{Le terminal méthanier au port de Grande-Anse}

En 1984, le gouvernement fédéral entreprit la construction du terminal maritime de Grande-Anse aux coûts de 25 millions de dollars. Ce port inauguré l'année suivante est la réponse aux pressions exercées antérieurement en faveur d'une relocalisation des réservoirs d'hydrocarbures de la zone portuaire de Chicoutimi. La décision paraît surprenante car, en 1981, une taxe sur l'entreposage des hydrocarbures a favorisé le transit des produits pétroliers par la route 175. Ainsi, la vocation initiale du port qui consistait au transbordement des hydrocarbures a évolué vers une fonction polyvalente orientée vers la manutention de produits forestiers, de marchandises générales, de vracs solides et liquides (le bois d'œuvre, les pâtes et papiers, le sel industriel, etc.). Depuis lors, ce port a connu une faible progression du tonnage manutentionné. Entre 1985 et 2005, 336000 tonnes ont été manipulées en moyenne avec une pointe de 461000 en 2001. En tout, 7 millions de tonnes ont été traitées à Grande-Anse en 21 ans d'opération.

\section{Ce port a connu une faible progression du tonnage manutentionné.}

C'est dans ce contexte qu'émerge le projet de construction d'un terminal méthanier d'importation de gaz naturel liquéfié (GNL) à proximité du terminal maritime de Grande-Anse. Le terminal méthanier de Grande-Anse permettrait la réception, le stockage, la regazéification et l'expédition vers les marchés de gaz naturel provenant de sources éloignées, de manière économique. Pour ce faire, le terminal comprendrait: des installations maritimes dédiées, des réservoirs de stockage de GNL, des installations de vaporisation du GNL, une usine d'extraction des composés lourds ainsi qu'une centrale de cogénération. Les marchés visés par le projet Grande-Anse sont, en ordre de priorité, le Québec, l'Ontario et le Nord-Est américain. À ce sujet, deux autres projets de ports méthaniers sont actuellement en processus d'évaluation environnementale au Québec, soit Cacouna et Rabaska.

Le promoteur du projet est la compagnie Énergie Grande-Anse, une entreprise de propriété régionale. Le projet serait réalisé en partenariat avec l'Administration portuaire du Saguenay qui est responsable du quai et des opérations maritimes. Cependant, un gazoduc devra être construit afin de relier le site de Grande-Anse au réseau de transport québécois. Le projet est en ce moment au stade du concept mais il a été soumis à un processus de consultation auprès de 25 intervenants de tous les secteurs d'activités. Le rapport du comité consultatif a été rendu public en décembre 2006 et celui-ci recommande d'aller de l'avant dans ce projet. Malgré le peu d'effets prévisibles sur le milieu humain, compte tenu de l'isolement du site, combien de mouvements de navires méthaniers y aura-t-il par années sur le Saguenay? N'oublions pas que ces navires transportent une matière dangereuse assujettie à des règles particulières de transport considérant le fait que la voie maritime du Saguenay fait partie $\mathrm{du}$ parc marin du Saguenay-Saint-Laurent. Ces enjeux environnementaux n'ont pas encore été abordés publiquement.

\section{Conclusion}

L'arrondissement de La Baie est un territoire à la croisée des chemins sur les plans économique et urbanistique. Trois sites importants sont en voie 
d'être réaménagés sous l'impulsion de promoteurs privés ou de l'administration municipale. Les projets qui seront mis de l'avant ainsi que leurs modalités d'implantation influenceront considérablement et durablement l'avenir et le paysage de l'arrondissement. Il appert de procéder à une analyse intégrée de l'aménagement et de l'urbanisme sur ce territoire. À l'heure actuelle, les projets semblent développés de manière indépendante, ce qui pose problème malgré leur valeur intrinsèque. Le processus de révision du plan et des règlements d'urbanisme en cours à la Ville de Saguenay devrait permettre de planifier les interventions territoriales à La Baie de manière cohérente sur la base d'une vision globale. Il est primordial de saisir les opportunités actuelles afin de relancer La Baie sur la voie du développement.

\section{Les projets semblent développés de manière indépendante, ce qui pose problème malgré leur valeur intrinsèque}

Toutefois, les projets envisagés soulèvent différents problèmes en matière de gouvernance. En effet, la gestion de certains dossiers par l'organisme Promotion Saguenay, alors que d'autres relèvent des services techniques de la municipalité, pourrait amener des difficultés de coordination. De plus, Promotion Saguenay est une organisation parapublique au sein de laquelle les agents économiques privés sont fortement représentés. Ce type de fonctionnement, qui s'apparente à un partenariat public-privé, n'est généralement pas souhaitable en matière d'aménagement urbain, considérant la nécessité d'arbitrer les intérêts en présence dans l'optique de l'intérêt collectif. Par ailleurs, la transparence et les démarches consultatives ne sont guère assurées selon cette méthode, comme le démontre le processus de mise en œuvre du terminal de croisières.

Malgré tout, La Baie semble appelée à profiter de la conjoncture pour façonner son avenir selon un concept axé sur la qualité du cadre de vie et les vocations touristique, commerciale et résidentielle. Mis à part le projet de terminal méthanier à GrandeAnse qui valorise la fonction industrielle, les projets à l'étude ou en cours de réalisation s'insèrent dans un nouveau créneau de développement pour l'arrondissement, c'est-à-dire les fonctions touristique et, possiblement, récréative. Certes, les nouveaux emplois créés ne seront peut-être pas aussi nombreux ou de la même qualité que ceux de l'ancienne papeterie d'ACI. Cependant, la mise en réseau du territoire à un degré plus élevé permettra de maintenir un milieu dynamique et animé au sein d'une agglomération dont les pôles d'emplois sont divers et répartis sur tout le territoire. Un milieu dans lequel les usages industriels sont présents mais regroupés en retrait de secteurs centraux revitalisés sur la base d'activités compatibles avec la qualité de vie et le tourisme.

\section{Notes et références}

1 Leblond, R. et Tremblay, L. (1977) Ville de La Baie, Plan directeur d'urbanisme, Leblond et Tremblay urbanistes, p. 144.

2 Le Quotidien, le 9 novembre 2006, Port d'escale à La Baie, Les séances d'information pour les citoyens débutent, par François Saint-Gelais, p. 6.

3. Brassard, H. (2001) Installations portuaires de PortAlfred, Compagnie $d u$ chemin de fer RobervalSaguenay: 75 ans d'ouverture sur le monde, La Baie, Alcan, p. 5.

4 Le quai Duncan, qui sera complètement aménagé en 1937-38, est destiné à l'importation de vracs secs (principalement la bauxite, l'alumine et le coke vert). Pour sa part, le quai Powell qui fera également l'objet de travaux importants en 1947-48, servira à l'importation des vracs liquides (l'huile, le caustique et le mazout) et l'exportation des marchandises finies et semi-finies vers l'étranger (lingot d'aluminium et rouleau de papier)

5 Compagnie Abitibi-Consolidated du Canada, (2005), Caractérisation de types Phase 1 et Phase 2, Cessation définitive des activités de l'usine Port-Alfred, Ville de Saguenay, Arrondissement de La Baie, Rapport final, Volume 1 de 4, DDH Environnement ltée, p. 3.

6 Notons que ce terrain sera décontaminé selon le niveau $\mathrm{C}$ de la grille du ministère du Développement durable, de l'environnement et de la faune du Québec. Ceci est la limite acceptable pour des terrains à vocations commerciale, industrielle et pour certaines activités récréatives (voir le site www.mddep.gouv.qc.ca). 